\title{
A Study on the Volatility of Housing Price in Seoul
}

\author{
Cha-Soon Choi $^{1}$ \\ Associate Professor 31020 Dept. of Real Estate Studies, Namseoul University, 91 \\ Daehakro, Seonghwan-eup, Seobuk-gu, Sheonan-si, Chungnam, Seoul, Korea \\ 1'chasoon59@nsu.ac.kr
}

\begin{abstract}
This study is empirical analysis on the volatility of housing price and the spillover effect, which was conducted using the EGARCH model for the time series data of key money deposit amount and the sale price of houses in Gangnam and Gangbuk of Seoul from January 2003 to April 2019. First, the result of the analysis showed that for the key money deposit and sale price model for Gangnam areas, EGARCH $(1,1)$ model that examines the asymmetric effect was more appropriate than the GARCH $(1,1)$ model. Second, it was found that there is the spillover effect from the key money deposit price in Gangnam to the sale price in Gangnam. However, there was no spillover effect on the sale price in Gangbuk and Seoul. A $1 \%$ increase in the key money deposit price in Gangnam led to a $0.158 \%$ increase in the sale price in Gangnam. Third, the volatility in house price in Gangnam and Gangbuk was affected more by the volatility from the previous month rather than the spillover effect of the fluctuation in the key money deposit price in Gangnam. It is necessary for the government to establish multiple demand and supply policies and dynamically control the market in order to stabilize the fluctuating housing market.
\end{abstract}

Keywords: Housing Price, Volatility, Spillover Effect, EGARCH Model, Housing Rental Price.

\section{Introduction}

As the volatility of asset increased since the global financial crisis, the volatility of housing price, which accounts for $60 \%$ of household assets, is also increasing. The housing market is instable as the increase rate of the housing price high as well as the volatility.[1] As the key money deposit price skyrocketed recently, the key money deposit to housing price ratio increased. And there is a careful forecast that the housing price will rise. Regarding the sharp rise in the housing price of Seoul, many people realize that the sudden rise of the key money deposit is affecting the housing price in Gangnam and Gangbuk that it will lead to an increased housing price. Therefore, this study aims to empirically analyze in-depth whether the increased volatility of key money deposit affects the volatility of housing price in order to suggest political implications. This study will use the EGARCH model to empirically analyze the volatility and spillover effect of housing price. Volatility means uncertainty, and in economics, it is an index that represents risks.

Foreign studies on volatility of housing price include Miller and Peng (2006) and Willcocks (2010). Domestic studies include Im (2006) and Kim and Jeong (2011). Miller and Peng (2006) used the GARCH model and the panel VAR model to analyze the relationship between the

Article history:

Received (March 18, 2019), Review Result (May 24, 2019), Accepted (June 29, 2019) 
time-variant volatility of quarterly housing prices in 277 metropolitans in the US and the macroeconomic variables. As the result, the GARCH model showed that the time-variant volatility effect was found in $17 \%$ of the 277 cities. Also, it was estimated that the volatility of housing price Granger-causes the GNP growth rate.[2] Willcocks (2010) used the GARCH model and the EGARCH model to analyze the volatility of quarterly housing price data from 13 regions in England. As the result, the ARCH effect was found in 7 out of 13 regions, and the asymmetric effect was shown in 6 regions.[3] Im (2006) used the GARCH, GJR, and EGARCH model to analyze the volatility of housing price in Seoul and 6 metropolitan cities in Korea. The result showed that there was asymmetry in the volatility but not leverage effect. Considering the asymmetry in apartments, the GJR or EGARCH model was suggested to be appropriate whereas the GARCH model was considered suitable for townhouses or singlefamily houses.[4] Kim and Jeong (2011) used the GARCH and EGARCH model to analyze the volatility of apartment prices from Seoul and 6 metropolitan cities. The result showed that the volatility of apartment price was largely affected by the news on the fluctuation of the apartment price. However, the size and durability of volatility differed by region.[5]

Whereas previous studies focused on empirically analyzing whether the volatility and spillover effect of housing price affect the housing market in a specific area, this study aimed to empirically analyze whether the volatility and spillover effect of key money deposit affect the housing market. Chapter 2 describes the analysis method, Chapter 3 shows the result of the analysis, and Chapter 4 summarizes the conclusion and implications.

\section{Theoretical Framework}

\subsection{EGARCH model}

The EGARCH model is a model developed by Nelson (1991) that eliminated the assumption where both parameters need to be a positive number to make the variation greater than zero. This model is suitable for analyzing an asymmetric effect.[6] This study will be using the EGARCH $(1,1)$ model, and the conditional variance formula is shown in (1).

$$
\sigma_{t}^{2}=\exp \left[\omega_{0}+\beta \log \left(\sigma_{t-1}^{2}\right)+\alpha\left\{\left|\frac{\epsilon_{t-1}}{\sigma_{t-1}}\right|-\sqrt{\frac{2}{\pi}}\right\}+\gamma \frac{\epsilon_{t-1}}{\sigma_{t-1}}\right\}
$$

The EGARCH $(1,1)$ model has modified the restrictions on parameters. In the conditional variance formula (1), $|\beta|<1$ makes the conditional variance formula stable. $\gamma$ is the parameter that measures the asymmetric effect. That is, when $\frac{\epsilon_{t-1}}{\sigma_{t}}<0$, then $\alpha-\beta$, and when $\frac{\epsilon_{t-1}}{\sigma_{t-1}}>0$, then $\alpha+\beta$ that the volatility in the EGARCH $(1, \Gamma)^{1}$ model reacts asymmetrically. If the volatility reacts greater to the news on increased price as in the housing market, the $\gamma$ value becomes a positive number. When $\gamma$ is a negative number, there is a leverage effect. With $\gamma \neq$ 0 , the shock becomes asymmetric. This study used the modified conditional variance formula from Eview 7.0 instead of the formula (1) used by Nelson (1991). The resulting EGARCH $(1,1)$ model is as shown in formula (2).

$$
\begin{gathered}
y_{t}=a+b y_{t-1}+\epsilon_{t} \\
\left.\sigma_{t}^{2}=\exp \left|\omega+\operatorname{slog}\left(\sigma_{t-1}^{2}\right)+\alpha\right| \frac{\epsilon_{t-1}}{\sigma_{t-1}} \mid+\gamma \frac{\epsilon_{t-1}}{\sigma_{t-1}}\right] \\
\text { However, } \omega=\omega_{0}-\alpha \sqrt{\frac{2}{\pi}}
\end{gathered}
$$




\subsection{Volatility and spillover effect model using the EGARCH model}

With the assumption of a one-sided spillover effect from the key money deposit in Gangnam to the housing price in Gangnam, Gangbuk, and Seoul, the Gangnam model was first estimated and then the Gangbuk and the Seoul models were estimated using the conditional variance data obtained from the Gangnam model. For this, a model shown in formula (3) is established.

$$
\begin{aligned}
& v_{1, t}=\beta_{1,1}+\beta_{1,2} \nu_{1, t-1}+v_{1, t} \\
& y_{2, t}=\beta_{2,1}+\beta_{2,1} \nu_{2,-1}+\beta_{2,3} \nu_{1, t-1}+v_{2,1} \\
& \left.\sigma_{1, t}^{2}=\exp \left[\alpha_{1,1}+\alpha_{1,2}\right] 0 \Omega \sigma_{1, t-1}^{2}+\alpha_{1,3}\left|\frac{\nu_{1, t-1}}{\sigma_{1 t-1}}\right|+\alpha_{1,4} \frac{\nu_{1, t-1}}{\sigma_{1 t-1}}\right] \\
& \sigma_{2, t}^{2}=\exp \left[\alpha_{2,1}+\alpha_{2,2} 10, \sigma_{2, t-1}^{2}+\alpha_{2,3}\left|\frac{\nu_{2 t-1}}{\sigma_{2, t-1}}\right|+\alpha_{2,4} \frac{\nu_{2 t-1}}{\sigma_{2, t-1}}+\alpha_{2,1}\left[0, \sigma_{1, t-1}^{2}\right]\right.
\end{aligned}
$$

Each variable and subscript for coefficients in (3) is used to differentiate each area. When calculating the spillover effect of apartment price from Gangnam to Gangbuk or Seoul, 1 refers to Gangnam whereas 2 refers to Gangbuk or Seoul. As this study aims to verify the one-sided spillover effect of key money deposit in Gangnam to the housing price in Gangnam, Gangbuk or Seoul, the analysis of the model only takes account of this matter. For Gangnam, the EGARCH $(1,1)$ model with verified validity was used. For Gangbuk or Seoul, the estimation was made by adding the primary variable $y_{1, t-1}$ of the volatility of the key money deposit in Gangnam to the average equation. The $\log$ value $\log \sigma_{1, t-1}^{2}$ of the conditional variance of Gangnam was added to the conditional variance equation.[7]

\section{Analysis result}

\subsection{Time series data and stability examination}

The data used in this study is from the monthly reports of KB Kookmin Bank on apartment sale price and key money deposit index. The rate of change was calculated using these monthly statistics. The monthly data from January 2003 to April 2019 were corrected considering the seasonal fluctuation, and the regions were divided into Gangnam, Gangbuk, and Seoul. The basic statistics of the data are as shown in $\langle$ Table 1$\rangle$.

Table 1.Basic Statistics on Fluctuation Rate of Housing Price

\begin{tabular}{|c|c|c|c|c|}
\hline & $\begin{array}{c}\text { Gangnam (Key money } \\
\text { deposit) }\end{array}$ & $\begin{array}{c}\text { Gangnam } \\
\text { (Purchase) }\end{array}$ & $\begin{array}{c}\text { Gangbuk } \\
\text { (Purchase) }\end{array}$ & $\begin{array}{c}\text { Seoul } \\
\text { (Purchase) }\end{array}$ \\
\hline Average & 0.351 & 0.373 & 0.303 & 0.348 \\
\hline $\begin{array}{c}\text { Standard } \\
\text { Deviation }\end{array}$ & 0.634 & 0.9687 & 0.798 & 0.834 \\
\hline Skewness & -0.430 & 2.2468 & 3.597 & 2.662 \\
\hline Kurtosis & 4.786 & 11.200 & 3.597 & 15.250 \\
\hline $\begin{array}{c}\text { Jarque-Bera } \\
\text { Statistic }\end{array}$ & $\begin{array}{c}31.94 \\
(0.000)\end{array}$ & $\begin{array}{c}710.41 \\
(0.000)\end{array}$ & $\begin{array}{c}3211.19 \\
(0.000)\end{array}$ & $\begin{array}{c}1449.70 \\
(0.000)\end{array}$ \\
\hline
\end{tabular}

Note) ( ) is of the significance level where the null hypothesis can be rejected.

The distribution of the sale price in Gangnam, Gangbuk, and Seoul skewed to the positive direction but not the key money deposit in Gangnam. The kurtosis was sharper than the normal 
distribution. The Jarque-Bera test result rejects the null hypothesis where the distribution of the volatility of housing price is a normal distribution at the $1 \%$ significance level, therefore, the GARCH model is required based on this variance. Meanwhile, the time series analysis takes stationary as the underlying premise. Thus, to examine the stationary of the variable, the augmented Dickey-Fuller (ADF) unit root test was performed.[8] The results are as shown in $<$ Table 2>, and the logarithmic time series data of each index were stable at $1 \%$ significance level.

Table 2. Unit root examination result

\begin{tabular}{|c|c|c|c|c|}
\hline & Gangnam (Key money deposit) & $\begin{array}{c}\text { Gangnam } \\
\text { (Purchase) }\end{array}$ & $\begin{array}{c}\text { Gangbuk } \\
\text { (Purchase) }\end{array}$ & Seou 1 (Purchase) \\
\hline T-value & -4.477 & -6.495 & -6.388 & 06.397 \\
\hline P-value & $0.000 * * *$ & $0.000 * * *$ & $0.000 * * *$ & $0.000 * * *$ \\
\hline
\end{tabular}

Note) 1. $\mathrm{p}<0.01^{* * *}, \mathrm{p}<0.05^{* *}, \mathrm{p}<0.1^{*} 2$. The lag for the test was determined to be 1 and included the constant term.

\subsection{Estimation by the model}

<Table 3> shows the estimation of housing price obtained with the EGARCH $(1,1)$ model to study the asymmetric response of volatility. The constant term of the equation was significant for Gangnam but not for Gangbuk and Seoul. As the $b$ value for each area was significant at the $1 \%$ level, it was shown that the sale price is affected by the sale price of the previous term. With the $\beta$ value from 0.69 to 0.98 , which is less than 1 , it was found that the conditional variance was stationary. Also, with the positive $\gamma$ value for all three areas, there was no leverage effect, but it could be concluded that there is an asymmetric effect from the shock at the $1 \%$ significance level. That is, an unexpected increase in the housing price could lead to greater volatility than the same amount of unexpected decrease in the housing price.

Table 3. EGARCH $(1,1)$ model estimates

\begin{tabular}{|c|c|c|c|}
\hline Statistic & Gangnam & Gangbuk & Seoul \\
\hline $\mathrm{a}$ & $0.0472(1.6669)^{*}$ & $-0.0078(-0.0986)$ & $0.1970(1.8135)$ \\
\hline $\mathrm{b}$ & $0.8060(31.3815)^{* * *}$ & $0.7794(17.1325)^{* * *}$ & $0.8022(24.7715)^{* * *}$ \\
\hline$\omega$ & $-0.0348(-0.8024)$ & $-1.4866(-11.3851)^{* * *}$ & $-0.0897(-1.9804)^{* *}$ \\
\hline$\alpha$ & $-0.0411(-0.6912)$ & $0.9934(9.8656)^{* * *}$ & $0.0224(0.3891)$ \\
\hline$\beta$ & $0.9868(210.125)^{* * *}$ & $0.6984(15.3713)^{* * *}$ & $0.9691(104.0086)^{* * *}$ \\
\hline$\gamma$ & $0.2209(5.7308)^{* * *}$ & $0.5610(6.9459) * * *$ & $0.2159(8.8720)^{* * *}$ \\
\hline $\mathrm{R}^{2}$ & 0.3739 & 0.5279 & 0.4568 \\
\hline $\mathrm{D} . \mathrm{W}$ & 2.1169 & 1.6547 & 1.9272 \\
\hline Log L & -122.66 & -55.99 & -94.97 \\
\hline
\end{tabular}

Note) 1. ( ) shows the z statistics. 2. $\mathrm{p}<0.01 * * *, \mathrm{p}<0.05^{* *}, \mathrm{p}<0.1 *$

With the assumption of a one-sided spillover effect from the key money deposit in Gangnam to the housing price in Gangnam, Gangbuk, and Seoul, the Gangnam model was first estimated. The Gangbuk and the Seoul models were estimated with the EGARCH $(1,1)$ model using the equation (3) for the conditional variance data obtained from the Gangnam model. And the result can be found in $\langle$ Table 4$\rangle$. 
Except for Gangnam and Gangbuk in the equation, all coefficients were found to be statistically significant. The $\alpha 2,2$ coefficient that is the AR (1) coefficient in the variance equation was less than 1 for Gangnam, Gangbuk and Seoul, and it can be said that the conditional variance is stationary.

The $\beta 2,3$ value, which explains the spillover effect of the key money deposit in Gangnam to the sale price in Gangnam, Gangbuk, and Seoul, was only significant for Gangnam at the 5\% level. A $1 \%$ change in volatility of the key money deposit in Gangnam led to a $0.158 \%$ increase in the sale price in Gangnam but did not affect the sale price in Gangbuk and Seoul. The $\alpha_{2,5}$ value that explains the spillover effect of volatility was found to be significant at the $1 \%$ level for Gangnam, Gangbuk, and Seoul. The $\alpha_{2,2}$ value that explains the volatility of the previous month was 0.687 for Gangbuk and 0.807 for Seoul, which was significant at the $1 \%$ level. The $\alpha_{2,5}$ coefficient was relatively smaller. From the long term aspect, the volatility of sale price in Gangnam, Gangbuk, and Seoul is affected more by the volatility of sale price in the previous month than the key money deposit of Gangnam.

Table 4. Spillover effect of volatility of key money deposit

\begin{tabular}{|c|c|c|c|}
\hline Statistic & $\begin{array}{c}\text { Key money deposit in } \\
\text { Gangnam } \rightarrow \text { Sale price in } \\
\text { Gangnam }\end{array}$ & $\begin{array}{c}\text { Key money deposit in } \\
\text { Gangnam } \rightarrow \text { Sale price in } \\
\text { Gangbuk }\end{array}$ & $\begin{array}{c}\text { Key money deposit in } \\
\text { Gangnam } \rightarrow \text { Sale price in } \\
\text { Seoul }\end{array}$ \\
\hline$\beta_{2,1}$ & $0.1452(1.043)$ & $0.015(0.192)$ & $0.174(1.695)^{*}$ \\
\hline$\beta_{2,2}$ & $0.822(20.8773)^{* * *}$ & $0.800(19.121)^{* * *}$ & $0.777(17.153)^{* * *}$ \\
\hline$\beta_{2,3}$ & $0.158(2.250)^{* *}$ & $0.041(0.951)$ & $0.094(1.410)$ \\
\hline$\alpha_{2,1}$ & $-0.505(-6.780)^{* * *}$ & $-1.406(-9.806)^{* * *}$ & $-0.859(-9.363)^{* * *}$ \\
\hline$\alpha_{2,2}$ & $0.909(37.609)^{* * *}$ & $0.687(15.516)^{* * *}$ & $0.807(17.590)^{* * *}$ \\
\hline$\alpha_{2,3}$ & $0.429(4.935)^{* * *}$ & $0.810(5.942)^{* * *}$ & $0.600(7.289)^{* * *}$ \\
\hline$\alpha_{2,4}$ & $0.377(5.90)^{* * *}$ & $0.721(6.490)^{* * *}$ & $0.489(6.737)^{* * *}$ \\
\hline$\alpha_{2,5}$ & $-0.080(-3.193)^{* * *}$ & $-0.089(-2.689)^{* * *}$ & $-0.077(-2.868)^{* * *}$ \\
\hline $\mathrm{R}^{2}$ & 0.379 & 0.538 & 0.467 \\
\hline $\mathrm{D} . \mathrm{W}$ & 2.199 & 1.704 & 1.939 \\
\hline Log L & -111.07 & -49.37 & -81.14 \\
\hline
\end{tabular}

Note) 1. ( ) shows the $\mathrm{z}$ statistics. 2. $\mathrm{p}<0.01 * * *, \mathrm{p}<0.05^{* *}, \mathrm{p}<0.1^{*}$

Meanwhile, $\alpha_{2,3}$ and $\alpha_{2,4}$ that explain the asymmetric effect of obtained information were 0.429 and 0.377 for Gangnam, 0.819 and 0.721 for Gangbuk, and 0.600 and 0.489 for Seoul. With both values being positive, there is an asymmetric effect, and this shows that an unexpected increase in housing price has a greater effect on volatility compared to the same amount of unexpected decrease in the housing price.

\section{Conclusion}

This study used the KB Kookmin Bank's housing price and key money deposit index from January 2003 to April 2019 to empirically analyze the volatility and spillover effect of price change in the key money deposit in Gangnam to the housing price in Gangnam, Gangbuk, and Seoul with the EGARCH $(1,1)$ model. In the key money deposit and sale price model for Gangnam, the $\gamma$ value that tests the asymmetric effect was found to be significant, and the EGARCH $(1,1)$ model was considered more suitable than the GARCH $(1,1)$ model.

As the result of studying the spillover effect of price change and volatility of the key money deposit in Gangnam to the housing price in Gangnam, Gangbuk, and Seoul, a 1\% rise in the volatility of key money deposit in Gangnam led to a $0.158 \%$ hike in the sale price in Gangnam. 
However, it did not affect the sale price in Gangbuk and Seoul. This is different from the general claim that the key money deposit leads the sale price. As the result of analyzing the spillover effect of the key money deposit in Gangnam on volatility, the volatility of sale price in Gangnam, Gangbuk, and Seoul was affected more by the volatility of previous month than the volatility of key money deposit in Gangnam. Also, the unexpected housing price shock had a greater impact than an unexpected decline in housing price.

This study is only limited to the housing market in Gangnam, Gangbuk, and Seoul that the spillover effect of the price change and volatility cannot be applied to all housing markets in Korea. However, political implications can be drawn from the following aspects. It was found that the volatility in the housing market is greater when the price rises rather than price declines, which is the opposite of the financial market. Therefore, it is necessary to pro-actively control the stability of the housing market when the housing price is on the rise. The data can be used as useful basic data for making investment decisions and establishing risk management standards. For robustness of the study, it will be necessary to add macro and micro data, not local data, to analyze the data from each region in Seoul as well as other provinces.

\section{Acknowledgements}

The funding for this paper was provided by Namseoul University, South Korea.

\section{References}

[1] S. J. Kwak and J. S. Lee, The Impacts of Public Policy on Housing Price Volatility Changes. Housing Studies Review. (2006), Vol.14, No.2, pp.175-194.

[2] N. Miller and L. Peng, Exploring Metropolitan Housing Price Volatility. Journal of Real Estate Finance and Economics. (2006), Vol.33, pp.5-18.

[3] G. Willcocks, Conditional Variences in UK Regional House Prices. Spatial Economic Analysis. (2010), Vol.5, No.3, pp.339-354.

[4] J. M. Lim, A study on the Volatility of Housing Sales Prices. Housing Studies Review. (2006), Vol.14, No.2, pp.65-84.

[5] J. H. Kim and J. H. Chung, A Studies on the Volatility of Housing Price Using GARCH, EGARCH Model. Korea Real Estate Academy Review. (2011), Vol.47, pp.367-383.

[6] D. B. Nelson, Conditional Heteroscedasticity in Asset Return: a New Approach. Econometrica. (1991), Vol.59, pp.347-370.

[7] S. K. Lee, The Spillover Effect of Price Chang and Volatility from Seoul Housing Mrket to Local Markets. Journal of Korea Planners Association. (2003), Vol.38, No.7, pp.81-90.

[8] D. Dickey and W. A. Fuller, Distribution of Estimates for Autoregressive Time Series with a Unit Root. Journal of the American Statistical Association. (1979), Vol.74, pp.427-431. 